\title{
Carré de Malberg et le droit constitutionnel de la Révolution française
}

Éric Maulin

\section{Q OpenEdition \\ 12 Journals}

Édition électronique

URL : https://journals.openedition.org/ahrf/583

DOI : 10.4000/ahrf.583

ISSN : 1952-403X

\section{Éditeur :}

Armand Colin, Société des études robespierristes

\section{Édition imprimée}

Date de publication : 1 juin 2002

Pagination : 5-25

ISSN : 0003-4436

\section{Référence électronique}

Éric Maulin, «Carré de Malberg et le droit constitutionnel de la Révolution française », Annales historiques de la Révolution française [En ligne], 328 | avril-juin 2002, mis en ligne le 11 mai 2006, consulté le 22 avril 2022. URL : http://journals.openedition.org/ahrf/583 ; DOI : https://doi.org/ 10.4000/ahrf.583

Ce document a été généré automatiquement le 22 avril 2022.

Tous droits réservés 


\title{
Carré de Malberg et le droit constitutionnel de la Révolution française
}

\author{
Éric Maulin
}

1 L'origine révolutionnaire des principes composant le fondement de notre ordre constitutionnel n'est plus aujourd'hui discutée, même si celui-ci semble parfois devoir être assoupli, adapté aux contraintes imposées par la renaissance d'un ordre juridique européen. Il n'en a pas toujours été ainsi. Sous la IIIe République, le droit constitutionnel révolutionnaire est resté assez peu étudié, objet de curiosité parfois mais de méfiance le plus souvent, identifié à une métaphysique de la souveraineté, à une conception absolutiste du pouvoir, dépassées, quelle que soit l'identité du titulaire de la puissance souveraine, le roi, le peuple ou la nation. Pour de nombreux publicistes, influencés par un positivisme diffus mais dominant, le droit révolutionnaire est une expression historique mais imparfaite du droit constitutionnel de l'État moderne. Il est un droit de l'âge métaphysique et absolutiste, au mieux considéré comme un point de départ qu'il convient de dépasser pour permettre à l'État de s'accomplir pleinement dans le régime parlementaire ${ }^{1}$.

Dans ce contexte, Raymond Carré de Malberg (1861 - 1935) fut l'un des rares auteurs à prendre le droit constitutionnel de la Révolution française au sérieux pour tenter de démontrer que ses principes étaient les fondations de l'État moderne. Son œuvre entière est consacrée à cette longue démonstration. Son maître ouvrage, la Contribution à la théorie générale de l'État, spécialement d'après les données fournies par le Droit constitutionnel français ${ }^{2}$, se présente comme une théorie juridique de l'État déduite des principes consacrés par la Révolution française. Par la suite, La loi, expression de la volonté générale, sous-titrée Étude sur le concept de loi dans la Constitution de 1875, se propose « de constater et d'établir, d'après les données fournies par le droit positif en vigueur, l'un des points capitaux du système constitutionnel français actuel (...) touchant la notion de loi et le pouvoir législatif ", à savoir l'influence exercée par "le concept énoncé dans le célèbre article6 de la Déclaration des droits de 1789 », qui 
définit la loi comme l'expression de la volonté générale ${ }^{3}$. Enfin, la Confrontation de la Théorie de la formation du droit par degrés avec les idées et les institutions consacrées par le droit positif français relativement à sa formation ${ }^{4}$, se propose de démontrer l'inapplicabilité de la théorie normativiste de Hans Kelsen et Adolf Merkl à la description de l'ordre juridique français, lequel ne repose pas sur une gradation des normes mais une hiérarchie des organes imaginée par les révolutionnaires français. L'œuvre entière, on le comprend, repose sur l'idée que les révolutionnaires, en particulier les constituants, de 1789 à 1791, n'ont pas seulement posé des règles de droit constitutionnel mais découvert les vrais principes de l'État moderne, dont Carré de Malberg peut alors, rétrospectivement, formuler la théorie.

Cette réception du droit révolutionnaire par Carré de Malberg s'opère toutefois dans un contexte, à la fois défini par l'objectif de son travail et les conditions de son existence, qu'il nous faut préciser.

D'une part, l'auteur est un juriste et non un historien. Par conséquent, le droit révolutionnaire est compris non comme une origine mais comme un fondement du droit constitutionnel moderne ; l'objet ultime visé par l'analyse n'est pas le droit du passé mais l'ordre juridique du présent ; la méthode de l'auteur n'est pas celle d'un archiviste mais d'un archéologue soucieux de mettre à jour les fondements. Cet aspect de l'œuvre apparaît nettement si l'on prête attention à la référence aux «données » du droit français ou du droit constitutionnel français. Ce qui est ici donné, c'est « du droit positif en vigueur ", mention importante pour un juriste positiviste qui ne définit le droit qu'en relation avec la volonté étatique qui le produit de manière continuée. Le droit est l'expression de la volonté de l'État. Quelle que soit la charge métaphysique d'une telle assertion, il faut ici considérer que le droit positif cesse simplement d'être du droit lorsqu'il n'est plus en contact avec la volonté de l'État. Le droit du passé, le droit abrogé, le droit caduc, le droit qui n'est plus irisé par la volonté de l'État, n'est plus du droit, c'est-à-dire qu'il n'a plus cette autorité qui caractérise sa force obligatoire sous menace de contrainte dans l'exécution par une autorité étatique. Il n'est plus l'expression de la puissance publique.

D'autre part, cependant, le droit positif consacré par l'État n'est considéré que comme une expression superficielle de principes qu'il révèle et qui le justifient. Disciple, sur ce point, de la doctrine positiviste et monarchiste allemande de C.F.Gerber, P.Laband et G.Jellinek, Carré de Malberg ne conçoit pas le droit comme un seul ensemble de règles posées mais comme un ensemble de principes fixes et sous-jacents dont les actes étatiques sont l'expression superficielle. L'ordre constitutionnel apparent repose sur un corps de principes qui le justifient et fondent le pouvoir. Le statut de ces principes est toutefois indéfini. Principes juridiques, ils ne sont pas posés mais paraissent exister en soi, comme un substrat juridique incréé qui est à l'origine de toute création. Il est manifeste que pour l'auteur, ces principes juridiques, comme la souveraineté nationale, la définition de la loi comme expression de la volonté générale, ont une valeur de vérité indépendante de la positivité des actes qu'ils justifient. Ni la souveraineté nationale ni l'identification de la loi à la volonté générale ne procèdent d'un acte constitutionnel, posé par l'État, mais ils sont bien plutôt la condition de possibilité de la formulation de tout acte positif. Ce n'est pas parce que ces principes sont posés qu'ils sont juridiques mais c'est parce qu'ils sont vrais qu'ils acquièrent une valeur juridique. Lorsque Carré de Malberg puise dans la Constitution de 1791 les principes qui lui permettent 
d'expliquer ensuite les fondements du pouvoir, il ne fait alors qu'expliciter la théorie de l'État, pour ainsi dire latente, contenue dans ces principes.

6 Mais la relation entre ces principes essentiels et la volonté de l'État, entre le vrai et le voulu, reste mystérieuse, inexpliquée. Elle révèle une tension entre un discours qui porte sur la seule légalité de l'ordre juridique considéré et un autre discours, parallèle, qui porte sur la légitimité de cet ordre et dont Carré de Malberg ne peut ni ne veut s'émanciper. En effet, le droit constitutionnel de la Révolution française est non seulement au fondement de l'ordre juridique français, mais à la racine de l'État moderne. Carré de Malberg juge que la Révolution fait accomplir au droit public (en général) un "grand progrès" ${ }^{5}$ en ce qu'elle consacre les vrais principes de l'État moderne. Pour cet Alsacien dont la famille, optant pour la nationalité française après 1870, avait choisi l'exil dans "la France de l'intérieur", le droit constitutionnel consacré par la Révolution française est non seulement une alternative historique à l'Ancien Régime français mais une alternative politique au droit monarchique allemand appliqué dans le Reichsland d'Alsace-Moselle ${ }^{6}$. La Contribution à la théorie générale de l'État ne se borne pas à systématiser le droit révolutionnaire comme fondement du droit français, formulant ainsi une théorie particulière de l'État français, mais procède à une analyse critique des fondements de l'ordre juridique allemand afin d'opposer au principe monarchique l'idéal de la souveraineté nationale. À bien des égards, l'œuvre de Carré de Malberg apparait comme une discussion critique de l'œuvre de Laband, professeur à l'Université impériale de Strasbourg de 1872 à 1917, date de sa mort 7, et auquel, peut-on dire, il succède en obtenant sa mutation, dès 1919, dans la nouvelle université républicaine de Strasbourg8.

7 La réception du droit constitutionnel de la Révolution française par Carré de Malberg est au centre d'intérêts intellectuels et biographiques qui en façonnent la compréhension. Loin de l'érudition historienne, dans une mythique retraite ou une méditation tranquille et sans danger, cette réception est au cœur d'une actualité politique et constitutionnelle à laquelle l'auteur, moins qu'un autre, ne peut échapper. Son œuvre reflète ces tensions plus qu'elle ne restitue le droit révolutionnaire dans sa vérité d'archive; elle justifie ou invalide un présent qui continue plus qu'elle ne représente un passé qui a cessé d'être.

L'avènement de l'État moderne

8 L'apport majeur de la Révolution française, selon Carré de Malberg, est d'avoir consacré le principe de la souveraineté nationale. Ce principe permet de penser une forme d'exercice de la puissance qui diffère aussi bien de la souveraineté monarchique que de la souveraineté populaire.

L'opposition de la souveraineté nationale et de la souveraineté monarchique

9 À la différence des auteurs qui ne voient dans ce principe qu'un nouvel habillage de l'absolutisme, une simple translation du pouvoir du monarque à la nation dans la forme inchangée d'une puissance souveraine, Carré de Malberg pense que la nature même du pouvoir s'en trouve affectée. Ce n'est pas un pouvoir de la même essence que le pouvoir monarchique qui se trouve transféré dans le Corps de la nation, mais le transfert de ce pouvoir opère une transformation de la nature même du pouvoir souverain. En effet, tandis que le monarque titulaire du pouvoir souverain est aussi le détenteur de son exercice, la nation, être anonyme et abstrait ${ }^{9}$, est le titulaire d'un pouvoir qu'elle ne peut pas exercer par elle-même, mais dont elle doit, par conséquent, "déléguer » l'exercice à un « représentant ». "L'œuvre capitale de la Constituante -écrit l'auteur -a 
consisté à séparer l'État de la personne royale et pour cela la Constituante fait intervenir la nation qu'elle oppose au roi comme le véritable élément constitutif de l'État, et par suite, comme seul légitime propriétaire de la puissance souveraine " ${ }^{10}$. Cependant il faut ajouter immédiatement que, selon le juriste alsacien, « la nation a été envisagée par la Constituante comme un ensemble indécomposable, comme un tout non morcelable et, par conséquent, aussi comme une unité globale, supérieure à ses membres individuels " ${ }^{11}$. C'est pourquoi le pouvoir dont est maintenant titulaire la nation se trouve immédiatement subordonné à la constitution qui organise l'État et donne compétence aux organes pour formuler la volonté nationale. Ainsi il n'y a pas seulement transfert mais transformation de la souveraineté.

Cette transformation a, pour l'auteur, une double signification. Premièrement, elle marque, la naissance du droit public moderne, c'est-à-dire la naissance d'un droit public institutionnalisé qui ne trouve plus son fondement dans la volonté psychologique d'un souverain physique mais dans la volonté juridique de l'Étatpersonne, incarnation de la volonté générale. La notion même de volonté souveraine se trouve ainsi transformée, comme le montre l'auteur lorsqu'il distingue entre le pouvoir constituant originaire, fait historique a-juridique et le pouvoir constituant dérivé, procédure juridique anhistorique. Cette transformation caractérise la naissance de l'État moderne. Avant la Révolution, l'État se confondait dans la personne du monarque ; avec la Révolution, le monarque disparaît et laisse place à l'État-nation souverain, à l'État comme personnification de la nation et la nation comme substrat de l'État. Deuxièmement, elle témoigne d'un progrès moral qui s'accomplit dans l'histoire, le droit moderne réalisant une limitation du pouvoir, lequel se trouve être toujours un pouvoir juridique ${ }^{12}$.

11 Pour comprendre la portée que Carré de Malberg reconnaît au principe de la souveraineté nationale, il faut s'arrêter, un instant sur le système que, selon lui, la Révolution met en place. Jusqu'à la Révolution, la souveraineté est entre les mains d'un monarque, lequel est souverain en ce qu'il est le titulaire originaire de sa puissance et ne dépend d'aucune volonté supérieure. Le monarque se trouve, par conséquent, dans une situation qui lui permet d'exercer immédiatement le pouvoir dont il est détenteur en propre. La monarchie absolue est une modalité du gouvernement direct. Au contraire, la Révolution, en transférant la souveraineté du monarque à la nation, c'est-à-dire, comme nous le verrons, à un être abstrait essentiellement distinct du peuple, neutralise le caractère absolu du pouvoir souverain en distinguant le titulaire anonyme du pouvoir suprême de son détenteur effectif, le représentant, qui ne fait que l'exercer en son nom. Ainsi se trouve supprimée l'existence d'un pouvoir absolu. L'opération réalisée par la Révolution consiste en un report du pouvoir du monarque sur la nation mais ce report consiste aussi en une transformation radicale de ce pouvoir. La portée de cet événement, qui modifie la nature même du pouvoir, dépasse évidemment la seule organisation "française" du pouvoir et se présente comme un modèle de l'organisation de l'État. Carré de Malberg écrit qu'en transférant ainsi le pouvoir du monarque à la nation, la Révolution «ne faisait que reconnaître, à sa manière, cette vérité théorique (...) à savoir que la puissance de domination étatique ne peut se concevoir que dans l'être synthétique et abstrait qui personnifie la collectivité nationale et qui n'est autre, en définitive, que l'État " ${ }^{13}$ Ce paradigme a une double valeur. D'une part, il rend compte d'une certaine organisation spécifique du pouvoir. En ce sens il a une valeur descriptive. Mais, d'autre part, et surtout, il s'impose comme le modèle de l'organisation de l'État, modèle qu'il convient d'imiter afin de réaliser l'État 
constitutionnel. La Révolution française marque un tournant dans la conception de l'État. L'auteur n'explicite pas cette thèse, et aucune philosophie de l'Histoire sousjacente ne vient la justifier, mais le sens des développements qu'il consacre au principe de la souveraineté nationale la suppose. À cet égard, il est tout à fait significatif que, dans son argumentation, l'opposition chronologique entre l'ancien principe monarchique et le principe révolutionnaire de la souveraineté nationale se métamorphose ensuite en une opposition synchronique entre le principe monarchique allemand et le système fondé par la Révolution. Manifestement alors le schéma révolutionnaire de l'organisation du pouvoir déborde la frontière géographique de son accomplissement historique pour s'imposer comme un véritable modèle, opposable à d'autres formes d'organisation du pouvoir.

De la monarchie absolue de l'Ancien Régime français Carré de Malberg rapproche en effet la monarchie limitée allemande au XIx ${ }^{\mathrm{e}}$ siècle, opposant ces deux figures de la monarchie pure à la monarchie apparente. Le principe monarchique est ici présenté comme le critère d'identification de la véritable monarchie. En effet, si dans la monarchie limitée le monarque n'exerce plus, comme dans la monarchie absolue, la puissance intégrale de l'État, «il n'en reste pas moins l'organe central et principal de l'État. Car c'est à lui qu'il appartient de mettre en mouvement l'activité étatique, en donnant l'impulsion aux organes autres que lui-même " ${ }^{14}$. Dans ce type de monarchie, actualisé par la Charte de 1814 ou la Constitution prussienne de 1850, le roi occupe une place prépondérante dans l'État. Il ne monopolise plus la totalité de la puissance étatique puisque au moyen d'une Charte ou d'une Constitution il a confié une part de l'exercice du pouvoir constituant et du pouvoir législatif à une ou plusieurs assemblées. Il n'est plus, en conséquence, le maître de la puissance étatique. Il ne gouverne plus selon le «bon plaisir ». Cependant il conserve d'importantes prérogatives et, surtout, ne les détient pas à titre de compétences dérivées de la constitution mais comme les reliefs d'une puissance originaire. Il détient, en particulier, un droit de sanction, de telle sorte que si le contenu de la loi est fixé par les chambres, le fondement de la force obligatoire de la loi est à rechercher dans l'exclusive volonté du monarque.

13 À la monarchie pure ainsi caractérisée Carré de Malberg oppose la monarchie apparente. Celle-ci n'est pas, à proprement parler, une véritable monarchie parce qu'elle ne repose pas sur un principe monarchique. La constitution n'y est plus octroyée par un monarque placé au-dessus d'elle mais, au contraire, le monarque est subordonné et constitué par une constitution placée au-dessus de lui. Quelle que soit alors l'importance de sa participation à l'exercice de la puissance, et notamment à la puissance législative, qu'il dispose d'un simple veto suspensif, comme dans la Constitution de 1791, ou d'un droit de sanction, comme dans la Constitution belge de 1831, le monarque est en principe subordonné à la volonté de la nation souveraine et ne peut en conséquence résister à une altération de ses compétences au moyen d'une révision. Il se trouve ainsi, comme l'ensemble des organes constitués, dans la situation d'une autorité précaire ${ }^{15}$. En somme, on le comprend, le critère de la distinction entre la véritable monarchie et la monarchie apparente réside dans la position du monarque par rapport à la constitution.

14 Fort de ce rapprochement entre la monarchie absolue et la monarchie limitée, toutes deux espèces du genre de la monarchie pure opposée à la souveraineté nationale, Carré de Malberg peut opposer le droit républicain français issu de la Révolution française au droit monarchique allemand issu de l'absolutisme. 

dériver en une forme absolutiste d'exercice du pouvoir. Carré de Malberg a soutenu ici qu'il fallait distinguer entre la souveraineté nationale - la bonne souveraineté nationale, peut-on écrire - et la mauvaise souveraineté populaire, cette distinction permettant seule, finalement, de comprendre que la Révolution n'a pas seulement transféré la souveraineté d'un titulaire à l'autre, mais qu'elle a inventé un mode d'organisation du pouvoir jusque-là inédit, appelé cependant à caractériser l'État moderne, lequel ne trouve son accomplissement qu'en lui.

L'opposition de la souveraineté nationale et de la souveraineté populaire souveraineté nationale, mais il a tenté en outre d'opposer cette dernière à la souveraineté populaire. L'argumentation qui fonde cette dernière opposition procède d'une assimilation structurelle de la souveraineté monarchique et de la souveraineté populaire. Il y a, écrit le juriste alsacien, une « essence commune de la monarchie et de la démocratie " ${ }^{16}$ qui réside dans la prétention d'une personne ou d'un groupe de personnes, le peuple, à exercer, en vertu d'un droit propre, le pouvoir souverain. Et c'est pourquoi finalement la démocratie aboutit aux mêmes résultats que la monarchie : la résorption de l'État dans la personne de son souverain et, finalement, la disparition de l'État. Dans le système monarchique, l'État se confond toujours avec la personne du souverain. Dans le système démocratique, l'État se dissout toujours dans le peuple c'est-à-dire dans la confédération des individus qui le composent. Monarchie et démocratie ne sont pas seulement "des formes de gouvernements ", mais "des façons d'être de l'État " ${ }^{17}$. Cependant ni la démocratie ni la monarchie ne permettent la réalisation de la véritable idée d'État. Seul l'État-nation réalise pleinement le concept juridique d'État, parce que seul il permet d'attribuer la souveraineté à un être collectif et abstrait, incapable d'exercer le pouvoir illimité dont il est le détenteur «Seule l'idée qui trouve son expression dans le principe français de la souveraineté nationale, permet de dégager pleinement le concept d'État, s'affirmant comme sujet permanent et distinct, c'est-à-dire à la fois, de ses organes, quels qu'ils soient, et des individus qui le composent à chacun des moments successifs de sa durée ${ }^{18}$.

Dans une version que Carré de Malberg attribue à Rousseau, le peuple est d'abord présenté comme une " masse de citoyens $~{ }^{19}$, « une collection inorganisée d'individus » ${ }^{20}$. Le principe de la souveraineté populaire consacre, ici, la confédération d'individus souverains ${ }^{21}$. En ce premier sens, le peuple est, en conséquence, incapable d'exprimer une volonté. Il est, comme dirait Hobbes, « une multitude désunie " ${ }^{22}$. La démocratie, que le principe de la souveraineté populaire consacre, aboutit à la désintégration de l'État: "l'État démocratique n'est pas un État véritable, car une confédération d'individus ne peut pas plus qu'une confédération d'États former un État" ${ }^{23}$. La comparaison est ici éclairante. Elle suggère que, comme dans l'ordre international une confédération d'États ne peut s'accorder qu'à l'unanimité, la démocratie appelle nécessairement comme son idéal mais aussi sa condition de possibilité - donc son impossibilité - l'unanimité des volontés des citoyens assemblés. C'est pourquoi Carré de Malberg pense, avec Bluntschli, que l'idée de la souveraineté du peuple est en contradiction avec "l'existence même de l'État " ${ }^{24}$. "Elle ne peut s'accorder avec aucune Constitution, pas même avec la démocratie absolue qu'elle prétend fonder : là aussi c'est l'assemblée ordonnée de la nation qui exerce la puissance publique, et non la foule atomisée ${ }^{25}$.

Annales historiques de la Révolution française, 328 | avril-juin 2002 

d'individus, à la différence d'une corporation, ne peut constituer une personne supérieure à celle des membres qui la composent. Le principe de la souveraineté populaire est, selon Carré de Malberg, indissociable de l'idée de contrat. Mais le contrat, constitutif d'une association, est en lui-même incapable de produire une personne morale supérieure aux individus qui s'associent parce qu'il est incapable de produire une puissance publique. L'association ne naît pas simplement de la volonté unanime des membres qui la composent, mais elle ne vit qu'à travers cette unanimité. Le contrat qui est à l'origine de l'association est, écrit Carré de Malberg, "un contrat qui se renouvelle sans cesse. Chaque homme, par cela seul qu'il continue de faire partie de la communauté nationale étatisée, concourt à tout instant à la formation de la nation et de l'État " ${ }^{26}$. Il est vrai qu'ailleurs Carré de Malberg écrit que l'objet du contrat social est « de créer, au sein de la société, une autorité publique, supérieure aux individus » ${ }^{27}$, c'est pourquoi chaque individu contractant «consent à une aliénation totale de sa personne à la communauté ». Mais, il ajoute immédiatement que « chaque membre est admis par tous les autres comme partie indivisible du tout, et par suite la volonté générale n'est elle-même pas autre chose que la somme numérique des volontés égales et associées ». Il en résulte selon lui « que la souveraineté a essentiellement son siège dans le peuple, c'est-à-dire dans les individus mêmes dont le peuple se compose, dans chacun des membres comptés un à un, de la masse populaire ${ }^{28}$. La souveraineté se trouve «divisée, émiettée par quotes parts personnelles, entre tous les membres ut singuli de la nation $" 29$.

Le contrat, l'association, ne peuvent faire naître une personne supérieure aux membres qui la composent parce qu'ils ne parviennent pas à produire une puissance publique, c'est-à-dire une puissance institutionnalisée dont le fonctionnement soit indépendant de la volonté des adhérents. C'est pourquoi la souveraineté y reste divisée et l'anarchie ainsi consacrée. Le système de la souveraineté populaire est ici absurde puisqu'il consacre une souveraineté des individus qui, par définition, est négatrice de la souveraineté de la collectivité unifiée. Si chaque individu est souverain, la décision collective ne peut se prendre qu'à l'unanimité des voix ou des volontés, exactement comme dans une confédération d'États, l'entente commune repose sur l'unanimité de la volonté des particuliers. Mais ce système est aussi irréaliste car « sur aucune question on ne saurait espérer obtenir une volonté absolument unanime de la part de tous les citoyens ${ }^{30}$. C'est pourquoi Rousseau est finalement bien obligé d'admettre le principe majoritaire, lequel expédient "nécessaire» est aussi «contradictoire» avec les prémisses de sa pensée, puisqu'il revient à admettre que des individus souverains se verront contraints par la majorité. En réalité le système majoritaire suppose que la soumission de chacun à la volonté de la majorité procède d'un « consentement donné d'avance " ${ }^{31}$. Fort de cette déformation des théories de Rousseau aux conséquences absurdes, l'auteur peut lui opposer la rationalité d'une souveraineté nationale qui combine harmonieusement l'unité de la souveraineté et la disparition de son exercice effectif.

On a fait justice d'une pareille conception qui, d'une part, déforme à un point surprenant la pensée de Rousseau, théoricien de l'intégration des individus dans la communauté et de l'universalité du peuple auteur de la loi ${ }^{32}$ et, d'autre part, transpose sur le lieu de la Révolution française une distinction/opposition de la souveraineté du peuple et de la souveraineté de la nation nées bien plus tard. Carré de Malberg en 
projette la signification sur la réalisation constitutionnelle du principe de la souveraineté populaire dans la Constitution de 1793. Cette dernière, pas plus que Rousseau, ne conçoit pourtant la souveraineté des individus, mais toujours celle de la collectivité du peuple, entendu comme unité. L'article7 de la Constitution de 1793 déclare clairement que "le peuple souverain est l'universalité des citoyens français ", les articles 25 à 27 de la Déclaration des droits consacrent pareillement l'idée que «la souveraineté réside dans le peuple; elle est une et indivisible ", "aucune portion du peuple ne peut exercer la puissance du peuple entier » et « tout individu qui usurperait la souveraineté (serait) à l'instant mis à mort " ${ }^{33}$. G.Bacot conclut en conséquence qu'« il n'y a donc pas dans la Constitution de 1793 de souveraineté individuelle qui puisse limiter la souveraineté du peuple ${ }^{34}$. Poursuivant plus loin son investigation, l'auteur ne découvre de conception individualiste de la souveraineté que chez les " enragés », et dans certains textes de Babeuf et des membres de la "Conjuration des égaux " ${ }^{35}$; mais Carré de Malberg ne cite ni ne s'appuie sur aucun des textes de ces courants minoritaires, dont la pensée n'a jamais déterminé la conception dominante du principe de la souveraineté populaire.

21 Afin de justifier la suprématie du principe de la souveraineté nationale, principe même du constitutionnalisme, Carré de Malberg est conduit à déformer les théories de la souveraineté monarchique et de la souveraineté populaire. Il est à noter cependant que cette déformation est ponctuelle. En effet, lorsque l'auteur s'attache à l'analyse des institutions des monarchies allemandes ou de la Confédération helvétique il n'a plus recours aux mêmes interprétations des principes qu'il oppose ici au principe de la souveraineté nationale. Progressivement d'ailleurs, au cours de son œuvre ultérieure, Carré de Malberg abandonnera progressivement les oppositions qu'il force ici.

La théorie révolutionnaire de l'organe d'État

22 C'est dans la forme de la représentation que s'accomplit l'État moderne. Conscient de l'importance de la théorie révolutionnaire de la représentation et de ses difficultés, Carré de Malberg a tenté de reformuler la théorie révolutionnaire de la représentation elle-même sous la forme d'une théorie de l'organe d'État.

23 L'un des apports majeurs de Carré de Malberg à la théorie de l'État est en effet d'avoir repensé le système constitutionnel de la représentation sous l'espèce d'une théorie de l'organe d'État, empruntant ainsi une terminologie d'abord imaginée par la doctrine allemande pour penser le statut du peuple dans l'État monarchique. Il faut toutefois préciser que l'auteur n'a pas voulu substituer une nouvelle théorie à l'ancienne, imparfaite, mais qu'il a entendu reformuler la pensée des révolutionnaires eux-mêmes. Il considère en effet que «si la théorie actuelle de l'organe juridique est de construction allemande, les matériaux en ont été fournis, en bonne partie, par les travaux et les discours des constituants français de 1791 le germe de cette théorie se trouve contenu dans leur concept du régime "représentatif et dans les définitions qu'ils ont données de ce régime " ${ }^{36}$. Et l'auteur ajoute encore "Dans ces conditions, il est permis de s'étonner que les auteurs français, reniant les origines françaises de la théorie de l'organe d'État, la fassent aujourd'hui passer pour une création exclusivement germanique. Cela est d'autant plus surprenant que les hommes de 1789 ont dégagé les éléments de cette théorie avec une fermeté et une précision que personne n'a dépassées depuis lors ». Toutefois il reconnaît aussi que la pensée des constituants n'apparait pas au premier abord parce qu'ils se sont servis d'un langage incorrect, usant du mot de « représentant » pour désigner le concept d'organes. Afin de découvrir 
la théorie révolutionnaire de l'organe d'État, il faut donc gommer le langage employé par les constituants et retrouver derrière les mots trompeurs ${ }^{37}$ les concepts et le système gravés dans la Constitution de 1791, s'attachant pour cela «non à ce qu'ils ont dit mais à ce qu'ils ont fait ».

Les origines allemandes de la théorie de l'organe d'État

La théorie de l'organe d'État est étroitement liée à la définition de l'État comme personne juridique, dans la mesure où elle permet de comprendre comment un être abstrait, une entité collective, peut " vouloir ", c'est-à-dire comment on peut lui prêter, lui imputer une volonté. Dans sa version la plus élaborée, celle de Jellinek, la théorie de l'organe consiste en une théorie de l'imputation. Cette définition a rempli une double fonction dans la doctrine monarchique allemande du XIX ${ }^{e}$ siècle. D'une part, elle a permis d'élaborer une science du droit public autonome, distincte aussi bien du droit privé, des théories patrimoniales du pouvoir, que de la science politique ou de la psychologie sociale et permettant de penser l'institution et la volonté de l'institution sans les confondre ni avec la propriété, ni le fait de la domination, ni la volonté psychologique du monarque. D'autre part, elle a permis de maintenir la monarchie authentique face à la montée du constitutionnalisme, en combinant le caractère constitutionnellement inconditionné de la puissance du monarque avec la nécessaire limitation de son exercice (théorie de l'autolimitation) ${ }^{38}$. Sous la plume des publicistes allemands, la théorie de l'organe d'État se présente à la fois comme une théorie générale, assujettissant tout exercice de la puissance au respect des règles constitutionnelles, et une théorie monarchiste réduisant la nation à sa représentation constitutionnelle, ce qui permet de sauver le caractère originaire de la puissance du monarque.

La théorie de l'organe d'État a incontestablement fasciné une grande partie de la doctrine juridique française de la fin du $\mathrm{xIX}^{\mathrm{e}}$ siècle et du début $\mathrm{du} \mathrm{Xx}^{\mathrm{e}}$ siècle, à la fois parce qu'elle permettait de surmonter certaines difficultés issues de l'interprétation de la représentation en terme de délégation et parce qu'elle présente un aspect fortement antidémocratique auquel certains juristes français sont particulièrement sensibles, au moment où l'institution du suffrage universel paraît menacer le fonctionnement du régime parlementaire ${ }^{39}$. Elle permet de rationaliser la théorie française de la délégationreprésentation et de relativiser l'influence du corps électoral afin de lutter contre les dérives $d u$ système représentatif ${ }^{40}$. Pour de nombreux auteurs, la théorie de l'organe s'inscrit dans le cadre d'une théorie générale de la monarchie limitée dans laquelle les chambres, appelées par le monarque, organisées par la constitution octroyée à participer à l'élaboration de la loi, ne jouent cependant que le rôle constitutionnel et, pour tout dire, subalterne, de conseiller du monarque, titulaire exclusif du droit de sanction. Cette limitation du rôle de la représentation repose elle-même sur l'idée que la nation, par elle-même, ne fait pas corps, elle n'est pas une réalité en soi, titulaire d'une souveraineté originaire dont elle déléguerait ensuite l'exercice à des organes, mais qu'elle n'existe juridiquement qu'au lieu et dans les limites constitutionnelles de sa « représentation ». Dans ces conditions on comprend que la « représentation » n'en est pas une, mais plutôt une production de la volonté « nationale».

Pour Gerber ou Laband, le corps des représentants de la nation ne tire pas son pouvoir de son origine élective mais d'une compétence constitutionnelle. À proprement parler, il ne représente personne, il n'est pas un re-présentant. Le mot de « représentation » n'est dès lors plus qu'une simple métaphore : il n'y a aucun rapport entre l'électeur et le député; le député n'exerce pas un droit appartenant à l'électeur; l'élection n'est 
qu'une fonction. Le député reste le fonctionnaire de la constitution octroyée par le roi. Les auteurs rejettent en effet "l'opinion grossièrement sensuelle qui regarde la population (...) comme équivalent à un peuple unifié " ${ }^{41}$. Ce n'est, en effet, que parce qu'il existe « un pouvoir politique unique que la multiplicité des membres se fond dans l'unité du peuple. Cette unité est celle du peuple qui constitue l'État" ${ }^{42}$. Sans représentation ou, plus généralement, sans organisation étatique, le peuple ne reste qu'une "multitude dissoute » une confédération de citoyens. Laband, dans le cadre de l'Empire allemand, insiste particulièrement sur cette dernière idée. Il pense que le peuple allemand «n'a pas de personnalité différente de l'Empire allemand et existant en dehors de lui » dans la mesure où il n'a pas de volonté ${ }^{43}$. Il n'existe, en conséquence, que dans la forme de sa représentation par un organe constitutionnel, ici le Reichstag ${ }^{44}$. Les membres de cet organe sont collectivement les représentants "du peuple tout entier » ${ }^{45}$. Toutefois il ajoute, logiquement, que la dénomination de représentants de l'ensemble du peuple, conventionnellement donnée aux membres du Reichstag, «n'a pas de signification juridique positive au sens juridique, les membres du Reichstag ne sont les représentants de personne leurs pouvoirs ne sont pas dérivés d'un autre sujet de droit ils reposent directement sur la Constitution de l'Empire " ${ }^{46}$. Laband est ainsi conduit à réduire considérablement la fonction du corps électoral, défini non pas comme le peuple mais « la somme des nationaux d'Empire ayant le droit de vote " ${ }^{47}$. Ce corps n'a cependant pas d'autre droit que ceux que la constitution lui reconnait sa participation à l'expression de la volonté étatique se limite et s'épuise dans le vote pour lequel il est convoqué. "Dès que le vote a eu lieu, toute participation, toute coopération, toute influence juridique de la collectivité du peuple, c'est-à-dire de tous les individus nationaux de l'Empire sur les décisions publiques, cessent du même coup " ${ }^{48}$. Dès lors il faut conclure que « le peuple et sa représentation sont juridiquement une seule et même chose" ${ }^{49}$. C'est pourquoi on peut conclure que la notion de représentation ne désigne "plus seulement comme autrefois un certain rapport entre le député et ses déléguants: il exprime l'idée d'un pouvoir consistant chez le représentant à vouloir et décider pour la nation ${ }^{50}$. Cependant si la nation ne veut que par le moyen de son organe de représentation, l'importance et la force juridique de sa volonté seront directement fonction de la place qu'occupe cet organe dans la constitution subordonné en monarchie mais, nous allons le montrer, principal en République.

Ainsi se manifeste bien l'ambiguïté de la théorie allemande théorie constitutionnaliste, elle assujettit le peuple à sa représentation, théorie monarchiste, elle confine la représentation dans une fonction seconde. La nation n'est qu'un organe de l'État parce qu'elle n'est pas le titulaire principal de la puissance.

Les origines révolutionnaires de la théorie de l'organe d'État

28 Carré de Malberg, dont l'œuvre est un peu postérieure aux auteurs ici évoqués, reprend et développe certains aspects de la théorie allemande de l'organe d'État. Toutefois, par un étrange retournement des concepts, il en fait le concept central d'une théorie de la souveraineté de la nation, exprimée par le canal de sa représentation. Ce faisant, il rend bien compte d'une tradition parlementaire française qui, jusqu'en 1958, ne concevait l'expression d'une volonté nationale qu'au lieu de sa représentation. Mais rend-il compte de la conception révolutionnaire de la représentation nationale

Par certains aspects la pensée de Carré de Malberg est proche des auteurs allemands ${ }^{51}$. Le maître strasbourgeois pense, en effet, que la nation ne se constitue comme unité et 
ne commence à vouloir qu'au lieu et dans la forme de sa représentation. Il reconnaît alors que la volonté des représentants est simplement attribuée à la nation : «L'acte de volonté de la personne organe est (...) attribué par le droit à la personne collective " ${ }^{52}$. Toutefois, dans le même temps, et paradoxalement en apparence, il refuse de réduire la nation à sa seule représentation et à la confiner ainsi au rang d'organe subordonné de l'État car il ne veut pas entériner une conception fictive de la représentation nationale ${ }^{53}$. Cette résistance aux conséquences de la théorie allemande se manifeste de deux manières.

D'une part, en dépit de la proposition souvent répétée selon laquelle la nation ne veut qu'au travers de ses organes, qu'il n'existe pas de volonté nationale antérieure à son expression organique, Carré de Malberg maintient cependant toujours l'idée d'une certaine représentativité de la représentation organique. «La notion d'organe, écrit-il en ce sens, suppose l'existence de certains liens entre le groupe et les individus qui veulent pour ce dernier ${ }^{54}$. Il ajoute encore, plus loin dans le texte : « Tout organe est nécessairement représentatif, il l'est plus ou moins, selon que ses attaches sont plus ou moins étroites ou plus ou moins étendues, mais il l'est toujours dans une certaine mesure. S'il ne l'était pas, il ne serait pas un organe du groupe " ${ }^{55}$. Sans doute l'exigence de représentativité ainsi formulée ne prend aucune forme juridique précise mais elle impose cependant à l'organe de s'inspirer de «l'esprit national » ou de ce que l'auteur, après Hauriou, appelle aussi le «bloc des idées incontestables ». À l'inverse « un étranger, dont la volonté serait imposée au groupe par une force venant du dehors, ne serait plus un organe de la collectivité mais un maitre " ${ }^{56}$. Il est difficile de préciser davantage la pensée de Carré de Malberg sur ce point car, par ailleurs, d'un strict point de vue juridique, il soutient que le représentant ou l'organe n'est lié par l'existence d'aucune volonté antérieure de sorte que la nation ne commence à vouloir non par l'intermédiaire de ses représentants mais par le moyen de son organe de volonté. On peut simplement conclure, là encore, que le maître de Strasbourg marque une fois de plus son attachement au principe de la souveraineté nationale comme l'expression d'une juste légitimité du pouvoir. L'expression de la volonté nationale coincide mystérieusement avec le substrat représenté.

31 D'autre part, Carré de Malberg rejette la théorie allemande de l'organe d'État parce qu'elle ne repose pas sur le principe de la souveraineté nationale mais sur le principe monarchique. Il pense que seul le principe de la souveraineté nationale est compatible avec la véritable théorie de l'organe parce que seul il autorise alors l'identification de la nation à l'État et non pas seulement à l'un de ses organes constitués: "la théorie de l'organe peut seule se concilier avec le principe de la souveraineté nationale ${ }^{57}$. Il ajoute que «dans le droit public moderne et spécialement dans le système juridique issu des principes dégagés par la Révolution française, la théorie de l'État corporatif devenue prédominante ne peut avoir, en soi et au fond, qu'une seule signification elle implique que l'État n'est pas autre chose que la personnification de la nation. L'État et la nation ne sont, sous deux noms différents, qu'un seul et même être. L'État, c'est la personne abstraite en qui se résume et s'unifie la nation Il est donc impossible d'opposer la personne étatique à la nation ${ }^{58}$.

En apparence ces propositions se concilient mal avec l'idée que les autorités législatives et constituantes sont les seuls organes de l'État, autrement dit que la nation ne commence à vouloir que par le moyen de son organe. Cette dernière idée ne semble pas, en effet, fondamentalement distincte de la conception allemande de l'organe. Dans 
les deux hypothèses, la nation ne veut que dans la forme et par le moyen de sa "représentation ». Toutefois il faut ici considérer que la réduction de la nation à un organe de l'État prend une signification très différente selon qu'elle est éclairée par le principe de la souveraineté monarchique ou le principe de la souveraineté nationale. En effet, les théories de la loi que l'on peut déduire de ces principes sont aux antipodes l'une de l'autre.

Dans la théorie monarchiste, ce n'est pas la nation mais bien le monarque qui est le titulaire principal de la puissance de l'État. Au contraire, les Chambres, représentants de la volonté nationale, ne détiennent qu'une compétence d'attribution dérivée de la constitution octroyée elles n'exercent qu'une compétence partielle, en collaboration avec le roi, dans un domaine restreint, celui de la loi. Dans un tel système, la loi votée par les Chambres et sanctionnée par le monarque n'est pas le principe ou la condition de toute activité étatique mais seulement une limite opposée à la puissance originaire et exclusive du monarque. Toutefois celui-ci conserve un pouvoir réglementaire initial et autonome qu'il peut exercer par ordonnance en vertu de sa puissance originaire dans les domaines qui ne sont pas affectés par la constitution au partage de compétence avec les Chambres. Par ailleurs, les théoriciens de la monarchie - Laband au premier chef accroissent considérablement le domaine de l'ordonnance autonome lorsqu'ils définissent matériellement et restrictivement la loi comme l'ensemble des droits relatifs aux sujets de l'État. Les Chambres ne sont alors compétentes que dans un domaine nettement circonscrit. La nation, représentée par les Chambres, n'est donc qu'un organe de l'État placé dans une position subalterne elle n'existe juridiquement que dans le champ borné par l'octroi. Tel est le système de l'État de droit, par opposition à l'État de police, qui protège les sujets de la volonté exclusive du monarque mais n'altère pas la puissance de principe que celui-ci détient.

Dans le système français, la nation est également un organe de l'État. Mais parce qu'un tel système repose sur le principe de la souveraineté nationale, il implique une toute autre conception de la loi. La nation souveraine ne se trouve plus placée dans une position subalterne, exerçant une compétence partielle et limitée mais elle est titulaire de toute la puissance de l'État. En conséquence, la volonté exprimée par les « représentants » de la nation, c'est-à-dire par l'organe législatif, est à l'origine de tous les actes de l'État. La loi n'est pas ici une simple limite à l'exercice de la puissance mais bien le principe et la condition de tout exercice de la puissance. Pour cette raison, nous l'avons vu, Carré de Malberg soutient que l'assemblée des représentants est le seul organe de l'État elle ne connaît, au-dessous d'elle, que des autorités subordonnées. Dans un tel système aucune autorité ne peut détenir une puissance ou une compétence concurrente ou autonome car il n'est pas d'autre source de la valeur juridique des actes étatiques que la volonté législative exprimée par l'organe d'expression de la nation. Celui-ci alimente ainsi chaque acte accompli par une autorité de l'État. Tel est le système de l'État légal dans lequel la compétence des autorités est conditionnée par la volonté exprimée par l'organe national.

Il résulte de la comparaison entre ces deux schémas que, dans le système allemand, la nation est juridiquement un organe subordonné exerçant une compétence partielle dans un domaine restreint. L'État existe indépendamment d'elle puisque le monarque reste le détenteur principal de la puissance et conserve un pouvoir réglementaire autonome et initial ${ }^{59}$. Les nationaux sont alors des sujets soumis à l'exercice d'une véritable domination. Le trait caractéristique de ce système est la sanction que le 
monarque imprime à l'expression de la volonté nationale pour la rendre obligatoire. Dans le système français, au contraire, la nation représentée est le seul organe de l'État, exerçant une puissance initiale et totale, de sorte qu'aucun acte étatique ne peut prendre naissance qu'en application d'une loi c'est-à-dire de la volonté nationale. L'État n'existe qu'au moyen de la volonté de la nation qu'il personnifie. Les nationaux sont ici des citoyens, la loi ne tirant sa force obligatoire que de son adoption par une Assemblée ou un Parlement c'est-à-dire de la volonté nationale. Dans le premier système, la loi est une limite à l'exercice de la puissance et la nation n'est qu'un organe de l'État, dans le second, la loi est la condition de l'exercice de la puissance, et l'État la personnification juridique de la nation.

36 Carré de Malberg ne se contente pourtant pas d'opposer ces deux systèmes, mais il veut démontrer, en outre, que le système français est supérieur au système allemand. La conception allemande de l'organe d'État déduite du principe monarchique réduit la nation au rang d'organe de l'État parce qu'elle ne repose pas sur le principe de la souveraineté nationale. Carré de Malberg pense cependant que l'on ne peut pas définir l'État autrement que comme la personnification juridique de la nation et que cette définition suppose nécessairement le principe de la souveraineté nationale. Il veut démontrer que ce système, formulé par les hommes de 1789 «avec une fermeté et une précision qui n'a pas été dépassée depuis lors », a été juridiquement réalisé par la Constitution française de $1791{ }^{60}$.

On peut cependant douter que le système décrit par Carré de Malberg puisse s'entendre comme il l'interprète. Il n'est pas certain que dès 1789-1791 la nation se soit entièrement confondue avec le seul organe de sa représentation. On remarquera, d'une part, que Carré de Malberg minimise considérablement le rôle juridique joué par le monarque dans la procédure législative sous la Constitution de 1791. On a pu reprocher à Carré de Malberg de ne tenir ici aucun compte de la reconnaissance de la qualité représentative au monarque et l'attribution corrélative qui lui est faite d'un droit constitutionnel de sanction. On ne peut soutenir que l'assemblée soit le seul organe de la nation, car si la nation est dans sa représentation, elle ne s'exprime complètement que dans la combinaison des volontés de l'assemblée et du roi, détenteur d'un veto suspensif. Selon l'articleII du préambule du TitreIII : «La Nation, de qui seule émanent tous les pouvoirs, ne peut les exercer que par délégation. La Constitution française est représentative les représentants sont le Corps législatif et le Roi " ${ }^{61}$. D'autre part, et plus généralement, la labilité du mouvement révolutionnaire et l'instabilité constitutionnelle de la France, interdisent de tirer ici des conclusions définitives. Mais surtout, ce qui frappe, à la lecture d'une œuvre tendue vers la démonstration de la supériorité de la conception révolutionnaire sur la conception allemande de l'organe d'État, est qu'elle finit par consacrer non la représentation de la nation souveraine mais la souveraineté de la représentation nationale, hypallage qui débouche sur le parlementarisme absolu que dénonçera l'auteur dans son ouvrage consacré à la IIIe République, dans lequel il démontre que si le fondement de la force obligatoire de la loi est l'expression de la volonté générale, cette expression est cependant monopolisée par un organe de production qui ne représente plus la nation réelle ${ }^{62}$. 


\section{NOTES}

1.Joseph BARTHÉLÉMY, Du régime parlementaire en France sous LouisXVIII et CharlesX, Paris, 1904, éd. Giard et Brière, Introduction.

2.Raymond CARRÉ DE MALBERG, Contribution à la théorie générale de l'État, 2tomes, Paris, 1920 et 1922, Librairie du Recueil Sirey (réimprimé par les éditions du CNRS en 1962).

3.Raymond CARRÉ DE MALBERG, La loi, expression de la volonté générale, Paris, 1931, Librairie du Recueil Sirey (réimprimé en 1984 par les éditions Economica). Les propos rapportés figurent dans l'avertissement de l'auteur en tête de l'ouvrage.

4.Raymond CARRÉ DE MALBERG, Confrontation de la Théorie de la formation du droit par degrés avec les idées et les institutions consacrées par le droit positiffrançais relativement à sa formation, Paris, 1933, Librairie du Recueil Sirey.

5.Contribution à la théorie générale de l'État (abrégé Contribution), t. II, p.171.

6.O.BEAUD, «La biographie de Carré de Malberg et sa pensée ", La science juridique française et la science juridique allemande de 1870 à 1918, Actes du colloque tenu à Strasbourg les 8 et 9 décembre 1995, dir. O.BEAUD et P.WACHSMANN, Annales de la faculté de droit de Strasbourg, 1997, $\mathrm{n}^{\circ} 1$.

7.P.-M.GAUDEMET, « Paul Laband et la doctrine française de droit public », R.D.P., 1989, pp. 957-979.

8.On se reportera ici au témoignage de R.REDSLOB, « R.Carré de Malberg, la personnalité », Relation des journées d'études en l'honneur de Carré de Malberg, Paris, 1966, Librairie Dalloz.

9.Contribution, t. II, p. 168.

10.Contribution, t.II, p. 169.

11.Contribution, t. II, p. 173 note 6.

12.Contribution, t.II, p. 171 « La Révolution (...) consacre cette vérité morale sur le terrain du droit en posant dans l'art. 3 de la Déclaration de 1789 cette notion capitale que le sujet juridique de la puissance souveraine, c'est proprement la nation (...) la Constituante fait accomplir au droit public un grand progrès qui consiste à distinguer désormais le souverain des personnages qui détiennent en fait la souveraineté ». Dans le même sens, Jean ROELS, Le concept de représentation politique au XVIII siècle français, éd.Nauwelaerts, Louvain-Paris 1969, 179 p. ; ici p. 93. M. DE LA BIGNE DE VILLENEUVE, Traité général de l'État, Paris 1929, Librairie du Recueil Sirey, 625 p. ; ici, t.I, p. 267, reprend la théorie de Carré de Malberg lorsqu'il insiste sur la portée de la substitution de la personne abstraite de la nation à la personne concrète du roi. Ainsi, on introduisait nécessairement une théorie de la représentation du pouvoir.

13.Contribution, t.II, p. 174, nous soulignons.

14.Contribution, t.II, p. 64. Et « d'autre part, c'est en lui pareillement que réside le pouvoir de décision définitive, par exemple le pouvoir de parfaire la loi après qu'elle a été votée par les Chambres ".

15.C'est, notamment, ce que relève JELLINEK, L'État moderne et son droit, trad. française G.Fardis, Paris, 1911 et 1913, éd. M. Giard et E. Brière, 2 volumes ici, Contribution, t.II, p. 65, p.174. L'analyse de Carré de Malberg semble d'ailleurs généralement admise.

16.Contribution, t.II, p. 184.

17.Contribution, t.II, p. 185 , note.

18. Contribution, t.II, pp. 185-186, note. 
19.Contribution, t. II, p. 152.

20.Contribution, t.II, p.334. En un sens analogue, HAURIOU, Principes de droit public, 2e éd., Paris 1916, p. 630, oppose la nation et le peuple. La nation, écrit-il, « c'est le groupe social pris en corps, avec toute son organisation sociale et politique, par conséquent avec ses classes dirigeantes et avec son gouvernement aussi bien qu'avec sa classe populaire ; c'est le groupe social en tant qu'exerçant une maîtrise sur tous ses éléments composants, en vertu de cette idée qu'un tout organisé exerce une sorte de domination sur chacun de ses organes, par le principe d'ordre que contient en elle-même l'organisation. En somme, la nation est une conception corporative et organique qui implique le principe d'ordre. Le peuple, ou demos, est une conception anticorporative et inorganique, c'est une collectivité, mais où tous les rangs et toutes les classes sont confondus, où il n'y a plus d'ordre établi, où il n'y a même plus d'organes permanents de gouvernement, où les mouvements de l'ensemble sont déterminés directement par l'impulsion de la majorité des individus assemblés, c'est la régression vers la horde, le troupeau, la foule, vers ce que l'on appelle en sociologie l'état grégaire. La souveraineté nationale, étant organique, est par cela même représentative, parce que les organes sont des représentants élus et que les principales manifestations de la souveraineté sont relatives, soit à l'élection de ses représentants, soit au fonctionnement des assemblées délibérantes qu'ils vont constituer ; la souveraineté nationale conduit donc au gouvernement représentatif. La souveraineté du peuple est au contraire, par instinct, hostile au gouvernement représentatif et elle conduit au gouvernement direct de l'assemblée du peuple ».

21. Contribution, t.II, p.165. Cette conception apparaît particulièrement bien dans le paragraphe consacré à la " théorie de la souveraineté du peuple », Contribution, t.II, pp. 152 et s. Mais elle traverse en réalité toute la Contribution. CARRÉ DE MALBERG reviendra sur cette conception première dans La loi, expression de la volonté générale. Mais il abandonnera alors l'opposition systématique de la nation et du peuple, remettant ainsi en question l'un des postulats essentiels de la Contribution. D'une manière générale, ainsi que nous le verrons, toute la théorie de la représentation patiemment construite dans la Contribution sur une certaine définition du mot « nation » est remise en question par l'évolution du sens du mot « nation ».

22.HOBBES, Léviathan, ch.XVIII, p.180, dans la trad. qu'en propose F.Tricaud.

23. Contribution, t.II, p. 165.

24.BLUNTSCHLI, Théorie générale de l'État, trad. Armand de Riedmatten, Paris, 1881, Librairie Guillaumin et Cie, p. 438. Dans le même sens, SALEILLES, « La représentation proportionnelle », R.D.P. 1898, pp.230-231 ; MESTRE, « Fondement juridique de l'élection proportionnelle ", Revue générale du droit, de la législation et de la jurisprudence, 1899, p. 445. Mais A. MESTRE révise son analyse dans « La notion de personnalité morale chez Rousseau ", R.D.P., 1902, pp. 447-468.

25.BLUNTSCHLI, Théorie générale de l'État, p. 438.

26. Contribution, t.I, p. 52.

27., t.II, p. 153.

28.Contribution, t.II, p. 154.

29. Contribution, t.II, p. 155.

30.Contribution, t.II, p. 155.

31.Contribution, t.II, p. 156.

32.Carré de Malberg assimile le principe de la souveraineté populaire à celui de la souveraineté de chaque individu considéré ut singuli avant la passation du contrat. Tout 
se passe alors comme si, le contrat ne pouvant faire naître une personne collective, il ne pouvait non plus produire une puissance publique, c'est-à-dire une puissance commune, la souveraineté de chacun persistant dans l'état de société. Rousseau n'a pourtant jamais soutenu une pareille thèse, en effet absurde. R. DÉRATHÉ,Jean-Jacques Rousseau et la science politique de son temps, Paris 1950, rééd. librairie Vrin, Paris 1988, 473 p., remarque que la souveraineté des individus, qui caractérise leur situation dans l'état de nature, ne se maintient pas après la passation du contrat et la constitution de la société civile, pas plus que le principe de la souveraineté populaire n'institue une confédération de citoyens. Bien au contraire, la passation du contrat entre les futurs membres du corps social et ce corps social en devenir est aussi le moment de la constitution d'une personne morale supérieure aux individus réunis. «À l'instant, au lieu de la personne particulière de chaque contractant, écrit Rousseau, cet acte d'association produit un corps moral et collectif » (Contrat social, Liv. I, ch. IV. (nous soulignons). La collectivité nouvellement créée est en réalité seule souveraine et dans cette nouvelle unité « les individus se perdent et disparaissent » (CASSIRER, « L'unité chez Rousseau ", Pensée de Rousseau, éd. Le Seuil, 1984, p.42).

33. Cité par Guillaumевасот, Carré de Malberg et la distinction entre souveraineté du peuple et souveraineté nationale, Paris 1985, éd. du C.N.R.S., p. 31.

34.BACOT, op. cit. p. 32.

35.ВАСОТ, op. cit., p. 50.

36.Contribution, t.II, pp. 297-298.

37.« La Constituante s'est donc exprimée d'une façon incorrecte, lorsqu'elle a continué à qualifier de représentative l'assemblée des députés » (Contribution, t.II, p.283). La terminologie employée est « vicieuse » (Contribution, t.II, p.366. Voir aussi p. 297). 38.Cf.V.GERBER, Grundzüge eines Systems des deutschen Staatsrechts, Leipzig, 1865, 2eéd., 1869, 3e éd., 1880 P.LABAND, Das Staatsrechts des Deutschen Reiches, 1reéd., 1876, 3 vol, 5eéd., 1911-1914, 4 vol., trad. française, Le droit public de l'Empire allemand, trad. française de C.Gandilhon et alii, 6 volumes, Paris, 1900-1904, éd. V.Giard et E.Brière G.JELLINEK, L'État moderne et son droit, trad. française G.Fardis, Paris, 1911 et 1913, éd. M.Giard et E.Brière, 2 volumes Allgemeine Staatslehre, Berlin 1914, Verlag von O. Hâring, 837 p. Pour une présentation générale, voir R.EMERSON, State and Sovereignty in Modern Germany, New-Haven, 1928, Yale U.Press 282 p. O. JOUANJAN, « Gerber et la constitution d'une science du droit public », La science juridique française et la science juridique allemande de 1870 à 1918, Colloque tenu à Strasbourg les 8 et 9 décembre 1995, publié dans les Annales de l'Université de Strasbourg, ${ }^{\circ} 1$ en 1997.

39.Voir notamment, N.SARIPOLOS, La démocratie et l'élection proportionnelle, Thèse, droit, Paris, 1899, éd. Rousseau, 2 vol. de 483 et 480 p. ; A.MESTRE, Les personnes morales et le problème de leur responsabilité pénale, thèse, droit, Paris 1899, éd.A.Rousseau, 360 p., et aussi, « Le fondement juridique de l'élection proportionnelle dans la démocratie », Revue générale du droit 1899, pp. 15 et s. ; L.MICHOUD, « La notion de personnalité morale », R.D.P. 1899 ; R.SALEILLES, « La représentation proportionnelle », vol IX, R.D.P. 1898, pp. 215-234 et 385-413; ORLANDO, « Du fondement juridique de la représentation politique ", R.D.P., 1895.

40.Partant du constat qu'il existe un écart fondamental entre la conception théorique de la représentation et sa mise en œuvre pratique. Voir, par exemple, H.Bouchet, $L a$ conception de la représentation dans la Constitution de 1875 et ses déviations postérieures, thèse, droit, Dijon, 1908, $180 \mathrm{p}$. 
41.JELLINEK, L'État moderne et son droit, t.II, p. 103.

42.JELLINEK, L'État moderne et son droit, t.II, p. 60. Définition qui répond à celle de Hobbes qui voit dans le peuple sans l'État une multitude dissoute. Mais Jellinek poursuit son raisonnement et soutient que, de la même manière, " il ne saurait y avoir de territoire que par l'État, le territoire étant en effet l'espace constituant le domaine de l'État ; considéré en dehors de l'État il ne représente plus qu'une partie de la superficie terrestre, politiquement sans importance ».

43.LABAND, Le droit public de l'Empire allemand, t.I, p. 443. Laband exprime très clairement la position constitutionnaliste en poursuivant : « il est donc hors d'état de donner mandat ou pleins pouvoirs ou d'exercer des droits ou des volontés par l'intermédiaire de représentants. Par conséquent, la dénomination de représentants de l'ensemble du peuple, donnée aux membres du Reichstag, n'a pas de signification juridique positive ; au sens juridique, les membres du Reichstag ne sont les représentants de personne ; leurs pouvoirs ne sont pas dérivés d'un autre sujet de droit; ils reposent directement sur la constitution de l'Empire ».

44.LABAND, op. cit., t.I, p. 437.

45. LABAND, op. cit., t.I, p. 440.

46.LABAND, op. cit., t.I, p. 443.

47.LABAND, op. cit., t.I, p. 445.

48. LABAND, op. cit., t.I, p. 444.

49.JELLINEK, op.cit., t.II, p. 279. La doctrine allemande ne distingue pas la nation de ses représentants. «Elle affirme que le titulaire originaire et unique de la souveraineté c'est l'État pris comme tel (...) Les gouvernants ne sont point les représentants de la nation, ils sont les organes de l'État »; DUGUIT, Traité, t. I, p. 612.

50.Contribution, t.II, p. 263.

51.Comme le souligne O.BEAUD, « La souveraineté » dans La Contribution à la théorie générale de l'État de Carré de Malberg, R.D.P., 1994, pp. 1251-1301.

52.Contribution, t.II, p. 306.

53.Contribution, t.II, p. 339.

54.Contribution, t.II, p. 294.

55.Contribution, t.II, p. 343, note.

56.Contribution, t.II, p. 294.

57. Contribution, t.II, p.345, note. L'auteur continue : « À ce point de vue encore, cette théorie apparaît - ainsi que l'observation en a déjà été faite - comme une émanation de la Révolution française, et point du tout comme une conception d'essence germanique ».

58.Contribution, t.II, pp. 331-332. C'est pourquoi on ne peut conclure, avec W.LEISNER, Le pouvoir constituant, thèse, droit, Paris, 1956, dact., p. 37, que la nation de Carré de Malberg est simplement l'État des auteurs allemands. Une telle interprétation néglige, en effet, la différence fondamentale qui distingue la nation représentée dans le système monarchique de la nation souveraine dans le système français.

59. Contribution, t.I, p.315: «Le monarque ne s'est dépouillé du libre exercice de la puissance législative qu'en ce qui concerne les règles dites de droit » mais « pour toutes les autres règles, il a conservé le pouvoir constitutionnel de les édicter par lui seul en forme d'ordonnance $"$.

60.Contribution, t.II, p. 297. 
61.Sur ce dernier point, la pensée de Carré de Malberg est ambiguë et finalement déterminée par son souci de démarquer le système révolutionnaire de l'organe de la théorie monarchiste allemande. D'une part, en effet, il reconnaît bien la qualité de représentant au roi en se référant clairement à l'article II du préambule du titreIII. En s'appuyant sur le caractère représentatif du roi il démontre que les constituants ont nettement dissocié la représentation de l'élection élaborant une conception objective (c'est-à-dire une théorie de l'organe) et non plus subjective de la représentation. Par ailleurs, il pense justement que le titre représentatif du monarque n'a pas seulement une portée « décorative et nominale » car « si le roi a été, en 1791, qualifié de représentant, ça été précisément pour le distinguer du simple fonctionnaire et pour marquer qu'à la différence de celui-ci, il avait, en certains cas du moins, le pouvoir de vouloir librement pour le compte de la nation »(Contribution, t.II, p. 270). La qualité représentative du monarque provient de l'étendue et de la nature même de ses compétences : c'est afin de pouvoir faire obstacle et « contrecarrer une décision de l'assemblée représentative » que le roi est " capable de vouloir pour la nation ». Cependant, d'autre part, tout en reconnaissant au monarque la qualité de représentant ou d'organe de l'État en ce qu'il peut vouloir de manière initiale, il conclut, en contradiction avec sa propre définition de la représentation ou de l'organe : « En résumé donc, étaient des représentants, d'après la Constitution de 1791 : 1.le corps des députés, dans l'ordre législatif ; 2.Le roi, dans l'ordre exécutif ». Il soutient en conséquence que le veto « simplement suspensif » qui est reconnu au roi n'implique aucune participation à l'exercice de la puissance législative. (Contribution, t.I, pp. 399-400. Contribution, t.I, p. 399, en note, il écrit : « Ce mot de sanction n'était que la conséquence d'une fiction que la Constitution de 1791 faisait intervenir dans une pensée de déférence et de ménagement vis-à-vis du monarque. Le caractère fictif de cette prétendue "sanction royale" ressort suffisamment des termes mêmes dans lesquels le fonctionnement de cette institution était développé »). Voir aussi : M. TROPER, La séparation des pouvoirs et l'histoire constitutionnelle française, Paris, 1980, L.G.D.J, pp. 30 et s. ; du même auteur, «Sur l'usage des concepts juridiques en histoire ", Annales E.S.C., 1992, n6, pp. 1171-1183; Ch. EISENMANN a réfuté cette interprétation concernant Montesquieu dans «L'Esprit des Lois et la séparation des pouvoirs », Mélanges en l'honneur de R.Carré de Malberg, Paris 1933, éd.Sirey, reprise dans les Cahiers de philosophie politique, nos 2-3, Bruxelles, 1985, pp.3-34 ; M. PERTUÉ. « L'inviolabilité du roi dans la Constitution de 1791 », 1791, la première Constitution française, Actes du colloque de Dijon, Paris, 1991, éd. Economica, pp.181-204.

62.La loi, expression de la volonté générale.

\section{RÉSUMÉS}

Le principe de la souveraineté nationale est selon Carré de Malberg au fondement de l'État moderne. Il implique une transformation de l'exercice même du pouvoir, par différence avec le principe monarchique et le principe de la souveraineté du peuple. Il repose sur une représentation de la nation souveraine que Carré de Malberg réinterprète cependant en lui 
appliquant la théorie monarchiste allemande de l'organe d'État, mais en essayant de démontrer que cette théorie est d'abord révolutionnaire.

Carré de Malberg and the Constitutional Law of the French Revolution. The principle of national sovereignty is according to Carré de Malberg a corner-stone of the modern state. It implies a transformation of the very exercise of power, unlike the principle of monarchy and that of the sovereignty of the people. It rests upon a representation of the sovereign nation which Carré de Malberg, however, reinterprets by applying to it the German monarchist theory of the organ of state, his aim being to show that this theory is first and foremost revolutionary.

INDEX

Mots-clés : révolution, représentation, souveraineté nationale, souveraineté populaire, principe monarchique, théorie de l'organe, monarchie, France, Allemagne

\section{AUTEUR}

\section{ÉRIC MAULIN}

Professeur de droit public. Université de Haute-Alsace. Faculté de sciences économiques et sociales 15 , rue des Frères Lumière 68093 Mulhouse Cedex 